\title{
Establishment and characterization of intraperitoneal xenograft models by co-injection of human tumor cells and extracellular matrix gel
}

\author{
YUQIN YAO ${ }^{1-3 *}$, YONGJUN ZHOU $^{2 *}$, XIAOLAN SU $^{2}$, LEI DAI $^{2}$, LIN YU $^{2}$, \\ HONGXIN DENG ${ }^{2}$, LANTU GOU ${ }^{2}$ and JINLIANG YANG ${ }^{1,2}$ \\ ${ }^{1}$ Research Centre for Public Health and Preventive Medicine, West China School of Public Health, \\ No. 4 West China Teaching Hospital, Sichuan University, Chengdu, Sichuan 610041; \\ ${ }^{2}$ State Key Laboratory of Biotherapy and Cancer Center, Collaborative Innovation Center for Biotherapy, \\ West China Hospital, Sichuan University, Chengdu, Sichuan 610041; \\ ${ }^{3}$ Guangdong Zhongsheng Pharmaceutical Co., Ltd., Dongguan, Guangdong 523000, P.R. China
}

Received October 22, 2014; Accepted July 21, 2015

DOI: $10.3892 / 01.2015 .3764$

\begin{abstract}
Establishing a feasible intraperitoneal (i.p.) xenograft model in nude mice is a good strategy to evaluate the antitumor effect of drugs in vivo. However, the manipulation of human cancer cells in establishing a stable peritoneal carcinomatosis model in nude mice is problematic. In the present study, the ovarian and colorectal peritoneal tumor models were successfully established in nude mice by co-injection of human tumor cells and extracellular matrix gel. In ovarian tumor models, the mean number tumor nodes was significantly higher in the experimental group (intraperitoneal tumor cell co-injection with ECM gel) compared with the PBS control group on the 30th day $(21.0 \pm 3.0$ vs. 3.6 $\pm 2.5 ; \mathrm{P}<0.05)$. The same results were observed in the colorectal peritoneal tumor models on the 28th day. The colorectal peritoneal tumor model was further used to evaluate the chemotherapy effect of irinotecan (CPT-11). The mean weight of peritoneal tumor nodes in CPT-11 treatment group was significantly less than that of the control group $(0.81 \pm 0.16$ vs. $2.18 \pm 0.21 \mathrm{~g} ; \mathrm{P}<0.05)$. The results confirmed the value of these i.p. xenograft models in nude mice as efficient and feasible tools for preclinical evaluation.
\end{abstract}

Correspondence to: Professor Jinliang Yang or Professor Lantu Gou, State Key Laboratory of Biotherapy and Cancer Center, Collaborative Innovation Center for Biotherapy, West China Hospital, Sichuan University, No. 1, Keyuan Road 4, Gaopeng Street, Chengdu, Sichuan 610041, P.R. China

E-mail: jinliangyang@scu.edu.cn

E-mail: goulantu@foxmail.com

${ }^{*}$ Contributed equally

Key words: extracellular matrix gel, intraperitoneal xenograft model, tumor, irinotecan

\section{Introduction}

Clinically, peritoneal carcinomatosis represents an extreme form of cancer progression with a poor prognosis that is common in ovarian, colon and stomach cancer (1). Ovarian carcinoma is the fifth cause of mortality from gynecological cancer in women. Furthermore, $\sim 70 \%$ of patients are diagnosed with peritoneal carcinomatosis at an advanced disease stage $(2,3)$. In colon cancer, $\sim 8 \%$ of patients have isolated peritoneal seeding at the time of the primary surgery, and $25 \%$ of patients with recurrence have been found to exhibit peritoneal cavity metastasis $(4,5)$. A previous study showed that systemic chemotherapy or intraperitoneal (i.p.) chemotherapy alone had no significant effects on patient survival in the clinic (6). Therefore, novel therapeutic approaches are required to improve the therapeutic effects on peritoneal carcinomatosis.

An i.p. xenograft experimental model in nude mice simulates the process of peritoneal dissemination in intra-abdominal cancers. This model could be used to verify the effects of novel therapeutic strategies for peritoneal carcinomatosis in preclinical trials. A number of i.p. carcinomatosis mouse models had been established to study the processes of peritoneal dissemination and to verify the effects of novel therapeutic approaches since the first report of subcutaneous (s.c.) heterologous growth of human ovarian cancer tissue in nude mice $(7,8)$. In a number of these studies, human tumor cells were used for s.c injection. In other studies, following growth of the tumor to a suitable size, according to the experiment employed, the tumor was removed and cut into pieces or cell clusters for i.p. injection into nude mice $(8,9)$. Certain models were established by surgical implantation of fresh human tumor tissues or in situ injection of tumor cells obtained from homogenized human tumor tissues $(10,11)$, whereas other models were established by i.p. injection of specific human cancer cell lines directly or using depth immunodeficiency mice $(12,13)$. However, these aforementioned methods were complicated. A low success rate, a long latency period to develop palpable i.p. carcinomatosis and the requirement for surgery are the main obstacles 
in developing these models. The easiest method is to implant the usual human cancer cell lines directly into nude mice by i.p injection. However, it is difficult to achieve heterologous growth of human cancer cell lines in the abdominal cavity of nude mice by direct i.p. injection due to the non-specific immune response in the nude mice and the change of survival microenvironment for the human cancer cells. In our previous study, $1 \times 10^{7}$ SKOV-3 and $6 \times 10^{6}$ HCT-116 cells were directly implanted in order to establish ovarian peritoneal carcinomatosis and colorectal peritoneal carcinomatosis (CRPC) in the nude mice by i.p. injection. However, only a small number of tumor nodes were found in the mouse abdominal cavities in the CRPC model group. Also, mice in the ovarian peritoneal carcinomatosis group demonstrated a long latency period to develop little palpable i.p. carcinomatosis (unpublished data). These issues called for a more efficient way to establish a peritoneal carcinomatosis experimental model in the nude mice.

Numerous studies have highlighted that the tumor microenvironment, which is composed of structural [extracellular matrix (ECM)] and soluble extracellular substances (cytokines, proteases and hormones), as well as cellular components, including tumor cells, inflammatory cells, fibroblasts, and vascular and lymphatic endothelial cells. The microenvironment has a critical function in the pathogenesis of tumors, including tumor invasion and metastasis (14-16). Therefore, it is hypothesized that the establishment of peritoneal carcinomatosis in nude mice by direct i.p. injection of human cancer cells could become easier if the peritoneal cancer cell microenvironment was accustomed to the growth of human tumor cells.

ECM gel is a temperature-sensitive and reconstituted nutritional compound that has been widely used in cell cultures, tumor cell migration, angiogenesis and other biological experiments (17-19). ECM gel is prepared from mouse Engelbreth-Holm-Swarm sarcoma, which contains laminin as a major component, and collagen type IV, heparan sulfate proteoglycans and entactin as minor components $(19,20)$. ECM gel has been shown to enhance the s.c. tumor growth of nude mice $(21,22)$. We hypothesized that the ECM gel could also promote the formation of tumor tissues in the abdominal cavity of nude mice, since the ECM gel, which itself was obtained from nude mice, could promote the growth of human tumor cells with no immune rejection.

In the present study, liquid ECM gel solution was used to suspend human ovarian cancer SKOV-3 and colon cancer HCT-116 cells. Two stable and simple peritoneal tumor models were established in nude mice through implanting these cell/ECM gel suspensions into the abdominal cavity of the mice with high success rates. Next, the i.p. chemotherapy effect of irinotecan (CPT-11), an antitumor drug that is generally used for metastatic colon or rectal cancer treatment, was evaluated using the colorectal i.p. xenograft nude mouse model established by this method.

\section{Materials and methods}

Drugs and cell culture. CPT-11 was purchased from Prizer Co. (Shanghai, China). The human ovarian cancer SKOV3 and colon cancer HCT-116 cell lines were obtained from the American Type Culture Collection (Manassas, VA, USA) and cultured in Dulbecco's modified Eagle's medium (Gibco Life Technologies, Carlsbad, CA, USA) supplemented with $10 \%$ heat-inactivated fetal calf serum, $2 \mathrm{mM}$ L-glutamine and $0.1 \mathrm{mg} / \mathrm{ml}$ amikacin. The cells were incubated in a humidified $5 \% \mathrm{CO}_{2}$ atmosphere at $37^{\circ} \mathrm{C}$ and harvested during the logarithmic growth phase. The cells were then resuspended in phosphate-buffered saline (PBS) or ECM gel for i.p. injection.

Animals. Female, 7-8-week-old, BALB/c athymic nude mice were purchased from the Laboratory Animal Center of Sichuan University (Chengdu, Sichuan, China) and acclimated for 1 week. The mice were caged in groups of five in an air-filtered laminar flow cabinet and fed with irradiated food and autoclaved reverse-osmosis treated water ad libitum. All procedures were performed under sterile conditions in a laminar flow hood. Animal experiments were conducted under guidelines approved by the Institutional Animal Care and Treatment Committee of Sichuan University (23), and the study was approved by the animal experiment ethics committee of West China Hospital, Sichuan University (Chengdu, China; approval no. 018).

Establishment of i.p. xenograft models in nude mice. The suspended human ovarian cancer SKOV-3 cells were injected into the abdominal cavities of 18 nude mice using a needle with a length of $16 \mathrm{~mm}$ and a diameter of $0.45 \mathrm{~mm}$. Specifically, $4 \times 10^{6}$ cells in $200 \mu \mathrm{l}$ PBS for the control group and $4 \times 10^{6}$ cells in $200 \mu 1$ cold liquid ECM gel (placed on ice early) for the experimental group were injected into 9 control mice and 9 experimental mice, respectively. In each group, 3 mice were randomly selected and sacrificed by cervical dislocation on the 10th, 25th and 30th days after i.p. inoculation. A total of $3 \times 10^{6}$ human colon cancer HCT-116 cells were suspended in $200 \mu \mathrm{l}$ PBS and $200 \mu \mathrm{l}$ cold liquid ECM gel for the control and experimental groups, respectively. Next, the solutions were injected into the abdominal cavities of the 18 nude mice as aforementioned. To investigate the progression and characteristics of the i.p. xenografts, three mice were randomly selected for sacrifice by cervical dislocation on the 7th, 14th and 28th days after i.p. inoculation.

Necropsy. The mice were dissected and observed macroscopically. Specifically, the sizes of the abdominal tumors and tumor nodes, and the locations of the tumors were recorded. Following this, the tumors were collected for histopathological analysis.

Histopathological analysis. To establish the colorectal peritoneal carcinomatosis (CRPC) model, the colonic tumor node, the apparent masses in the abdominal cavity and the mesentery were stripped down on the 7 th, 14th and 28th days, respectively, and then frozen with optimal cutting temperature (OCT) compound. The 5- $\mu \mathrm{m}$ frozen sections were obtained from the optimal cross-sectional surface of the masses. The sections were then stained with hematoxylin and eosin (H\&E) and examined under light microscopy. For the ovarian peritoneal carcinomatosis model, when the mice were sacrificed on the 25th and 30th days, the apparent masses in the abdominal cavities of the nude mice were 
Table I. Dynamic progression of i.p. xenografts of human ovarian and colorectal cancer in nude mice at different time-points post-i.p. injection.

\begin{tabular}{|c|c|c|c|}
\hline Cell line & Day & Tumor nodes in ECM group, $\mathrm{n}^{\mathrm{a}}$ & Tumor nodes in PBS group, $\mathrm{n}^{\mathrm{a}}$ \\
\hline \multirow[t]{3}{*}{ SKOV-3 } & 10 & $6.3 \pm 2.5^{\mathrm{b}}$ & $1.0 \pm 1.0$ \\
\hline & 25 & $17.7 \pm 2.1^{\mathrm{b}}$ & $3.3 \pm 1.5$ \\
\hline & 30 & $21.0 \pm 3.0^{\mathrm{b}}$ & $3.6 \pm 2.5$ \\
\hline \multirow[t]{3}{*}{ HCT-116 } & 7 & $8.0 \pm 2.6^{\mathrm{b}}$ & $1.7 \pm 0.8$ \\
\hline & 14 & $13.0 \pm 2.0^{\mathrm{b}}$ & $3.0 \pm 1.0$ \\
\hline & 28 & $>50^{\mathrm{b}}$ & $5.0 \pm 1.5$ \\
\hline
\end{tabular}

${ }^{\mathrm{a}}$ Mean \pm standard deviation. ${ }^{\mathrm{b}} \mathrm{P}<0.05$ vs. PBS group. i.p., intraperitoneal; ECM, extracellular matrix; PBS, phosphate-buffered saline.

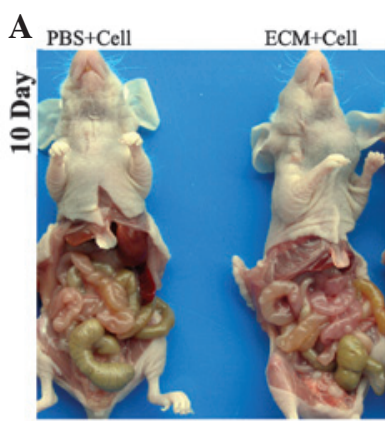

D H\&E

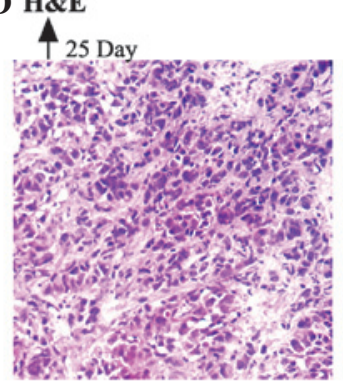

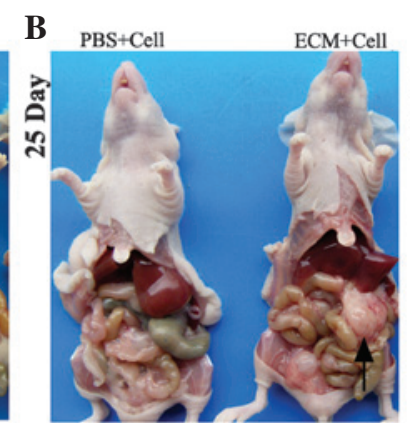

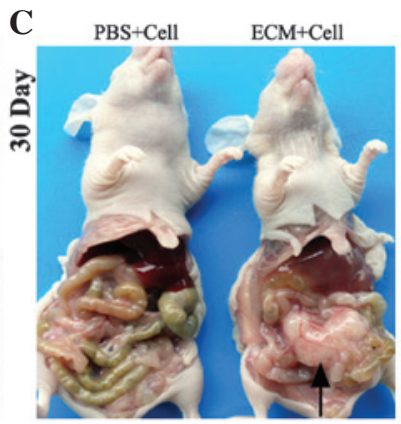

E
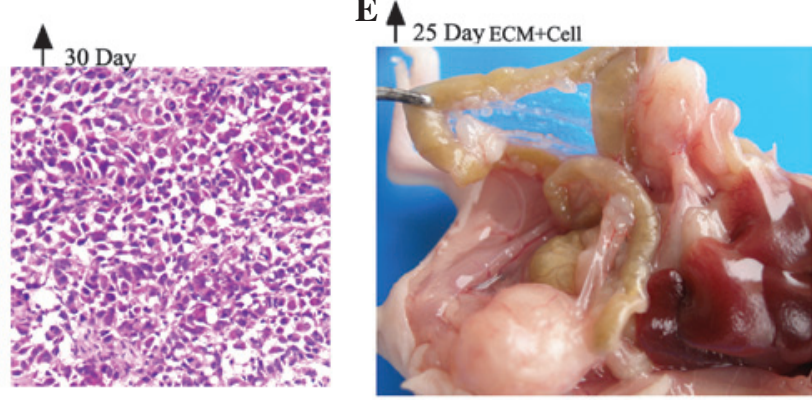

Figure 1. Macroscopic and microscopic observation of ovarian intraperitoneal xenografts in nude mice sacrificed on the 10th, 25th and 30th days. Macroscopic and representative images of mice from the PBS and ECM groups sacrificed on (A) the 10th day, (B) the 25th day, and (C) the 30th day, with black arrows indicating the representative tumor nodule. (D) Microscopic observation of H\&E staining of frozen sections (x100 magnification) of the tumor nodules indicated by the black arrows on the 25th and 30th day, respectively. (E) Magnified image showing the peritoneal cavity of the mouse from the ECM group, as indicated by the black arrow, on the 25th day. ECM, extracellular matrix; PBS, phosphate-buffered saline; H\&E, hematoxylin and eosin.

stripped down and frozen with OCT compound. The frozen section and H\&E analysis were performed as aforementioned.

In vivo antitumor evaluation of CPT-11 by the CRPC nude mice model. A CRPC therapeutic nude mice model was established and treated in a similar fashion. Briefly, $3 \times 10^{6}$ HCT-116 cells were injected into the abdominal cavities of nude mice using a needle that was $16 \mathrm{~mm}$ in length, with a diameter of $0.45 \mathrm{~mm}$. The mice were randomly divided into groups of five for control and experimental conditions after acclimation for 1 week (day 0 ). The mice of the test group were injected with i.p. CPT-11 at a dose of $10 \mathrm{mg} / \mathrm{kg} / \mathrm{day}$, whereas the mice in the control group were injected with i.p. normal saline (NS) at the same volume as the test group on days 0,4 and $8(24,25)$. The body weights of all mice were measured every 3 days. The mice were sacrificed by cervical dislocation on the 21st day, when the tumor nodes and the weights of the peritoneal tumors were measured.
Statistical analysis. Data are expressed as the mean \pm standard deviation. An unpaired two-tailed t-test was performed to calculate significance differences using SPSS software (version 17.0; SPSS, Inc., Chicago, IL, USA). P $<0.05$ was considered to indicate a statistically significant difference.

\section{Results}

Dynamic progression of the ovarian i.p. xenografts. For the ovarian i.p. xenograft mice (Fig. 1), at 10 days post-i.p. injection, 3 mice from each group were randomly selected and sacrificed. The mean number of tumor nodes was higher in the experimental group $(6.3 \pm 2.5)$ than the control group $(1.0 \pm 1.0)$ (Table I). No ascites or macroscopic tumors were found in the control or experimental subjects (Fig. 1A).

At 25 days post-i.p. inoculation, no evident abdominal distension was observed in either model. However, all the 

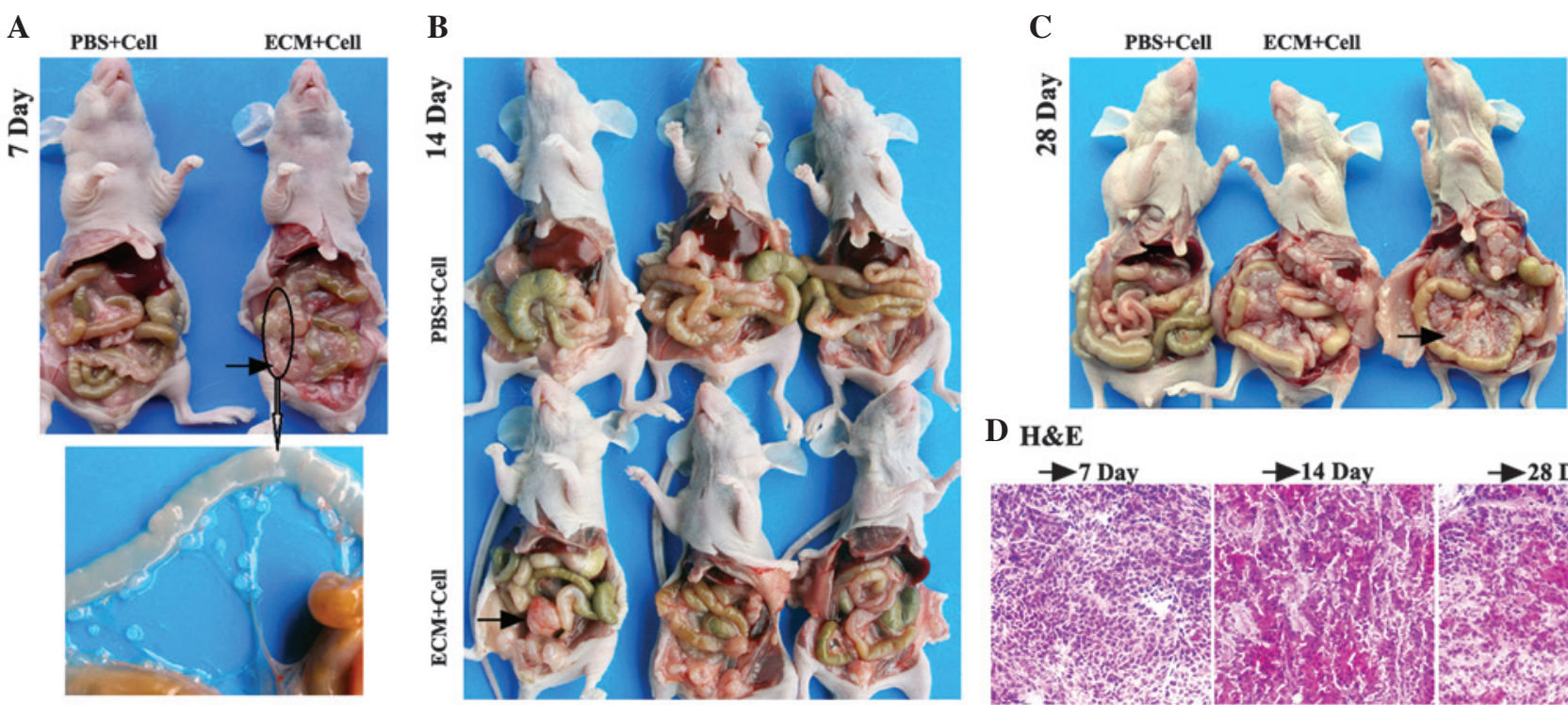

\section{H\&E}

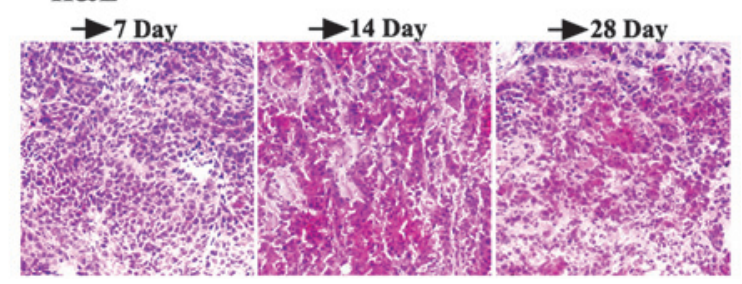

Figure 2. Macroscopic and microscopic observation of colorectal intraperitoneal xenografts in nude mice sacrificed on the 7th, 14th and 28th days. Macroscopic and representative images of mice from the PBS and ECM groups sacrificed on (A) the 7th day, (B) the 14th day and (C) the 28th day, with black arrows indicating the representative tumor nodules. (D) Microscopic observation of the H\&E staining of frozen sections (x100 magnification) of the tumor nodules indicated by the black arrows on the 10th, 14th and 28th days. ECM, extracellular matrix; PBS, phosphate-buffered saline; H\&E, hematoxylin and eosin.

mice in the experimental group $(n=9)$ had developed palpable tumor masses in the abdominal cavities. A total of 3 mice from each group were randomly selected and sacrificed. The mean number of tumor nodes was higher in the experimental group (17.7 \pm 2.1$)$ than the control group $(3.3 \pm 1.5)$ (Table I). The largest tumor node in the experimental group was up to $17 \mathrm{~mm}$ in diameter, whereas in the control group, it was $<7 \mathrm{~mm}$. The distribution of the tumor nodes was widespread, with locations that included the mesentery, the omentum, the intestinal surface, the retroperitoneum and around the pancreas (Fig. 1B). Partial enlargement of the abdomen was also observed in the experimental subjects (Fig. 1E).

The remaining mice $(n=6)$ were sacrificed at 30 days post-i.p. inoculation. Despite the bigger palpable tumor masses observed in the abdominal cavities of the experimental subjects, the general characteristics of the abdomens in these two groups were not significantly different compared with the mice sacrificed on the 25th day. Again, the mean number of tumor nodes was higher in the experimental group (21 \pm 3$)$ compared with the control group $(3.6 \pm 2.5)$ (Table I). The tumor nodes in the experimental subject had developed up to $24 \mathrm{~mm}$ in diameter (Fig. 1C).

Dynamic progression characteristics of colorectal i.p.xenografts. For the colorectal i.p. xenograft mice, 3 mice from each group were randomly chosen and sacrificed at 7 days post-i.p. inoculation. No ascites or macroscopic tumors were observed in the mice of either group. However, several small tumor nodules of $<1 \mathrm{~mm}$ diameter were found on the mesentery of one experimental subject (Fig. 2A). The mean numbers of tumor nodes are shown in Table I.

A total of 3 mice from each group were randomly chosen and sacrificed at 14 days post-i.p. inoculation. Tumor masses were observed in the abdominal cavities of two experimental subjects (Fig. 2B). The mean numbers of tumor nodes are shown in Table I.
Marked abdominal distension was observed in the mice of the experimental group at 28 days post-i.p. inoculation. Furthermore, two of the mice had developed numerous bloody ascites. Meanwhile, as expected, marked abdominal distension and bloody ascites were not found in the control subjects (Fig. 2C).

Histopathological analysis. In order to determine whether the intra-abdominal nodes observed were actually tumorous, $\mathrm{H} \& \mathrm{E}$ staining of the frozen ovarian and colorectal i.p. xenograft tumor node sections were performed. Microscopic analyses of the specimens were conducted to determine the tumorous identity. For the CRPC model, the tumor nodes on the colons of the mice of the experimental group sacrificed on the 7th day, the apparent masses in the abdominal cavities of the nude mice sacrificed on the 14th day and the masses on the mesenteries of the nude mice sacrificed on the 28th day were stripped down for the H\&E staining of frozen sections. For the ovarian peritoneal carcinomatosis model, when the mice were sacrificed on the 25th and 30th days, the apparent masses in the abdominal cavities of the nude mice in the experimental group were stripped down for the H\&E staining of frozen sections. The microscopic observations of the H\&E staining (x100 magnification) of these abdominal cavity masses are shown in Fig. 1D and 2D. The staining analysis confirmed that these intra-abdominal nodes in the ovarian and colorectal i.p. xenografts were tumor tissue.

In vivo antitumor evaluation of CPT-11 in the CRPC nude mice model. To determine whether the i.p. xenograft nude mouse models could be efficient as preclinical therapeutic models, an additional experiment using the colorectal i.p. xenograft nude mouse model was performed to evaluate the i.p. chemotherapy effect of CPT-11. CPT-11 was most active against this i.p. xenograft nude mouse model at a dose of $10 \mathrm{mg} / \mathrm{kg} / \mathrm{day}$, administered 3 times every 4 days (Fig. 3). The size of the 

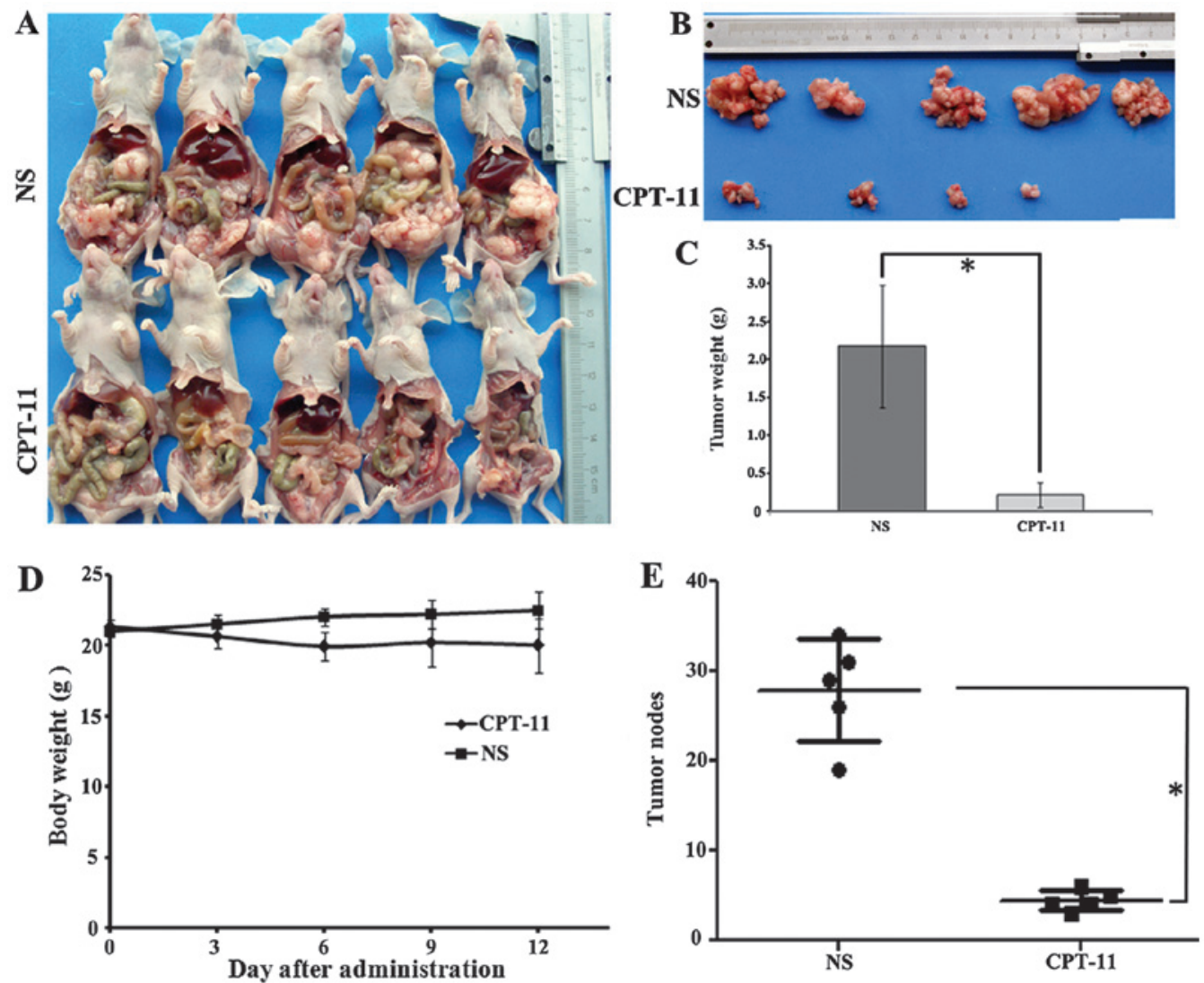

Figure 3. Antitumor evaluation of CPT-11 performed in a colorectal peritoneal carcinomatosis nude mouse model in vivo. (A) Macroscopic observation of mice sacrificed on the 21st day in the NS control and CPT-11 groups. (B) Macroscopic observation of total tumor nodules of the mice in the NS control and CPT-11 groups. (C) Total weight of peritoneal tumor nodules in the NS control and CPT-11 groups. (D) Body weights of mice were monitored every 4 days. (E) Numbers of tumor nodes in the NS control and CPT-11 groups. "P<0.05. CPT-11, irinotecan; NS, normal saline.

tumor nodes in the CPT-11 treatment group was significantly smaller than that of the NS group (Fig. 3A and B). The total weight of the peritoneal tumor nodes in the CPT-11 treatment group $(0.81 \pm 0.16)$ was significantly less than that of the NS group $(2.18 \pm 0.21 ; \mathrm{P}<0.05$; Fig. $3 \mathrm{C})$. All the tumor nodes in the two groups were counted and marked as shown in Fig. 3E. The mouse body weights were monitored every 4 days, and there was a significant difference in weight between the NS and CPT-11 groups $(\mathrm{P}<0.05$; Fig. 3D).

\section{Discussion}

Peritoneal carcinomatosis is a secondary cancer that occurs when cancer cells metastasize from other areas of the body and implant into the abdominal cavity. Conventional chemotherapy methods, including systemic chemotherapy or i.p. chemotherapy alone, exhibit no significant effects on survival in the clinic (26). Novel drugs and chemotherapy approaches are required to improve the therapeutic effects of peritoneal carcinomatosis. Nude mouse i.p. xenograft experimental models simulate the process of peritoneal dissemination in intra-abdominal cancers and could be used to verify the therapeutic effects of novel drugs and treatment approaches for pre-clinical evaluations. The easiest method for establishing the i.p. xenograft experimental model in nude mice is to directly implant the human cancer cell lines into the abdominal cavity of nude mice. However, the heterologous growth of human single cancer cells in the abdominal cavity of nude mice is difficult to achieve, with obstacles such as the residual non-specific immune function in nude mice that are difficult to overcome (27). One previous study indicated that a tumor tissue mass could grow well in the abdominal cavity of nude mice, but would require surgery and wounding of the mice (28). In the present study, to enable the growth of the cells and the progression into a tumor tissue mass, liquid ECM gel was used to suspend the human ovarian cancer SKOV-3 cells and the human colon cancer HCT-116 cells. Two nude mouse peritoneal tumor models were established by i.p. injection to implant these ECM gel cell suspensions into the abdominal cavities of the nude mice (Figs. 1 and 2).

Liquid ECM gel $\left(2-8^{\circ} \mathrm{C}\right)$ functions in a similar manner as PBS to suspend cells sufficiently. When the cell suspensions are injected into the abdominal cavities of nude mice, the PBS cell suspension is distributed in the form of single cells within the abdominal cavity, whereas the ECM gel cell suspension is distributed in the form of a cell mass due to the fact that the ECM gel forms into a jelly at $37^{\circ} \mathrm{C}$ (29). ECM gel is a mixture of ECM proteins produced by a mouse sarcoma cell line in vivo. The ECM gel is rich in laminin and collagen IV, which could stimulate tumor cell adhesion and motility. Therefore, cells in the ECM jelly could adhere to the surface of the mouse intestines and peritoneum more easily than when in the form of single cells. As they are surrounded by an outer layer of ECM gel, cells in the ECM jelly could more 
easily avoid the attacks from natural killer cells, monocytes and macrophages.

ECM gel also provides a good microenvironment for tumor growth. Liquid ECM gel cell suspension undergoes thermal-activated polymerization at $20-40^{\circ} \mathrm{C}$ when injected into the abdominal cavities of nude mice to form a reconstituted jelly anchoring a high density of tumor cells. Cells that are in contact with each other within the ECM gel are conducive to cells growth and signal transduction. The ECM jelly, including laminin as a major component, and collagen type IV, heparan sulfate proteoglycans and entactin as minor components, is also a rich store of angiogenic and tumor growth factors (30). For example, certain fragments of laminin-1, collagen IV and other matrix proteins can increase angiogenesis, tumor growth and metastasis $(31,32)$.

Currently, the CRPC mouse model, established by the injection of the mouse colon cancer CT-26 cell line into the abdominal cavity of BALB/c mice, is widely used to evaluate the i.p. chemotherapy effect of novel i.p. chemotherapy strategies (33). However, the application of a CRPC nude mouse model established using human cancer cells is rare. In the present study, an additional experiment was performed using the colorectal i.p. xenograft nude mouse model to evaluate the i.p. chemotherapy effect of CPT-11. Our previous study using the ovarian i.p. xenograft nude mouse model established by this method was also performed to evaluate the anticancer effect of T-DM1, an antibody drug conjugate (34). These results also confirmed that the i.p. xenograft nude mouse models established in this method are efficient and available preclinical therapeutic models for intra-abdominal cancers.

\section{Acknowledgements}

The authors are grateful to Mr. Shijie Zhou, Dr Ping Tang, Dr Rui Zhou and Mr. Cong Ma of the State Key Laboratory of Biotherapy and Cancer Center/Collaborative Innovation Center for Biotherapy (West China Hospital, Chengdu, China) Sichuan University for providing excellent technical assistance. This study was supported by the National Science and Technology Major Projects of New Drugs (grant no. 2012ZX09103301-037).

\section{References}

1. Kusamura S, Baratti D, Zaffaroni N, Villa R, Laterza B, Balestra MR and Deraco M: Pathophysiology and biology of peritoneal carcinomatosis. World J Gastrointest Oncol 2: 12-18, 2010.

2. Muñoz-Casares FC, Rufián S, Arjona-Sánchez Á, Rubio MJ, Díaz R, Casado Á, Naranjo Á, Díaz-Iglesias CJ, Ortega R, Muñoz-Villanueva MC, et al: Neoadjuvant intraperitoneal chemotherapy with paclitaxel for the radical surgical treatment of peritoneal carcinomatosis in ovarian cancer: A prospective pilot study. Cancer Chemother Pharmacol 68: 267-274, 2011.

3. Guardiola E, Delroeux D, Heyd B, Combe M, Lorgis V, Demarchi M, Stein U, Royer B, Chauffert B and Pivot X: Intra-operative intra-peritoneal chemotherapy with cisplatin in patients with peritoneal carcinomatosis of ovarian cancer. World J Surg Oncol 7: 14, 2009.

4. Macrì A, Saladino E, Bartolo V, Adamo V, Altavilla G, Mondello E, Condemi G, Sinardi A and Famulari C: Peritoneal carcinomatosis of colorectal origin. World J Gastrointest Oncol 2: 98-101, 2010.

5. Sadeghi B, Arvieux C, Glehen O, Beaujard AC, Rivoire M, Baulieux J, Fontaumard E, Brachet A, Caillot JL, Faure JL, et al: Peritoneal carcinomatosis from non-gynecologic malignancies: Results of the EVOCAPE 1 multicentric prospective study. Cancer 88: 358-363, 2000.
6. Yan TD, Stuart OA, Yoo D and Sugarbaker PH: Perioperative intraperitoneal chemotherapy for peritoneal surface malignancy. J Transl Med 4: 17, 2006.

7. Davy M, Mossige J and Johannessen JV: Heterologous growth of human ovarian cancer. A new in vivo testing system. Acta Obstet Gynecol Scand 56: 55-59, 1977.

8. Mei LJ, Yang XJ, Tang L, Hassan AH, Yonemura Y and Li Y: Establishment and identification of a rabbit model of peritoneal carcinomatosis from gastric cancer. BMC Cancer 10: 124, 2010.

9. Massazza G, Tomasoni A, Lucchini V, Allavena P, Erba E, Colombo N, Mantovani A, D'Incalci M, Mangioni C and Giavazzi R: Intraperitoneal and subcutaneous xenografts of human ovarian carcinoma in nude mice and their potential in experimental therapy. Int J Cancer 44: 494-500, 1989.

10. Ward BG, Wallace K, Shepherd JH and Balkwill FR: Intraperitoneal xenografts of human epithelial ovarian cancer in nude mice. Cancer Res 47: 2662-2667, 1987.

11. Fidler IJ: Critical factors in the biology of human cancer metastasis: Twenty-eighth G.H.A. Clowes memorial award lecture. Cancer Res 50: 6130-6138, 1990.

12. Santoro L, Boutaleb S, Garambois V, Bascoul-Mollevi C, Boudousq V, Kotzki PO, Pèlegrin M, Navarro-Teulon I, Pèlegrin A and Pouget JP: Noninternalizing monoclonal antibodies are suitable candidates for 125 I radioimmunotherapy of small-volume peritoneal carcinomatosis. J Nucl Med 50: 2033-2041, 2009.

13. Xu Y, Silver DF, Yang NP, Oflazoglu E, Hempling RE, Piver MS and Repasky EA: Characterization of human ovarian carcinomas in a SCID mouse model. Gynecol Oncol 72: 161-170, 1999.

14. Zhong L, Roybal J, Chaerkady R, Zhang W, Choi K, Alvarez CA, Tran H, Creighton CJ, Yan S, Strieter RM, et al: Identification of secreted proteins that mediate cell-cell interactions in an in vitro model of the lung cancer microenvironment. Cancer Res 68 : 7237-7245, 2008

15. Gout $\mathrm{S}$ and Huot J: Role of cancer microenvironment in metastasis: Focus on colon cancer. Cancer Microenviron 1: 69-83, 2008.

16. Fidler IJ, Kim SJ and Langley RR: The role of the organ microenvironment in the biology and therapy of cancer metastasis. J Cell Biochem 101: 927-936, 2007.

17. Xu X and Prestwich GD: Inhibition of tumor growth and angiogenesis by a lysophosphatidic acid antagonist in an engineered three-dimensional lung cancer xenograft model. Cancer 116: 1739-1750, 2010 .

18. Nicosia RF and Ottinetti A: Modulation of microvascular growth and morphogenesis by reconstituted basement membrane gel in three-dimensional cultures of rat aorta: A comparative study of angiogenesis in matrigel, collagen, fibrin and plasma clot. In Vitro Cell Dev Biol 26: 119-128, 1990.

19. Kleinman HK and Martin GR: Matrigel: Basement membrane matrix with biological activity. Semin Cancer Biol 15: 378-386, 2005.

20. Carey DJ, Todd MS and Rafferty CM: Schwann cell myelination: Induction by exogenous basement membrane-like extracellular matrix. J Cell Biol 102: 2254-2263, 1986.

21. Akbasak A, Toevs CC and Laske DW: Reconstituted basement membrane (matrigel) enhances the growth of human glioma cell lines in nude mice. J Neurooncol 27: 23-30, 1996.

22. Ishii E, Greaves A, Grunberger T, Freedman MH and Letarte M: Tumor formation by a human pre-B leukemia cell line in scid mice is enhanced by matrigel and is associated with induction of CD10 expression. Leukemia 9: 175-184, 1995.

23. Wang X, Duan X, Yang G, Zhang X, Deng L, Zheng H, Deng C, Wen J, Wang N, Peng C, et al: Honokiol Crosses BBB and BCSFB and inhibits brain tumor growth in rat $9 \mathrm{~L}$ intracerebral gliosarcoma model and human U251 xenograft glioma model. PLoS One 6: e18490, 2011.

24. Sumitomo M, Koizumi F, Asano T, Horiguchi A, Ito K, Asano T, Kakizoe T, Hayakawa M and Matsumura Y: Novel SN-38-incorporated polymeric micelle, NK012, strongly suppresses renal cancer progression. Cancer Res 68: 1631-1635, 2008.

25. Nagano T, Yasunaga M, Goto K, Kenmotsu H, Koga Y, Kuroda J, Nishimura Y, Sugino T, Nishiwaki Y and Matsumura Y: Synergistic antitumor activity of the SN-38-incorporating polymeric micelles NK012 with S-1 in a mouse model of non-small cell lung cancer. Int J Cancer 127: 2699-2706, 2010.

26. Micames C, Jowell PS, White R, Paulson E, Nelson R, Morse M, Hurwitz H, Pappas T, Tyler D and McGrath K: Lower frequency of peritoneal carcinomatosis in patients with pancreatic cancer diagnosed by EUS-guided FNA vs. percutaneous FNA. Gastrointest Endosc 58: 690-695, 2003. 
27. Sasiak AB, Sebesteny A, Hrivnak G and Lloyd DH: Experimental dermatophilosis in murine models of immunodeficiency. Rev Elev Med Vet Pays Trop 46: 263-269, 1993.

28. Zeng QL, Chu ZH, Zhou K and Luo XJ: Effect of Endostatin and SU6668 combined with 5-FU on human colon cancer xenograft in nude mice. Zhonghua Wei Chang Wai Ke Za Zhi 11: 376-378, 2008 (In Chinese).

29. Frisk T, Rydholm S, Andersson H, Stemme G and Brismar H: A concept for miniaturized 3-D cell culture using an extracellular matrix gel. Electrophoresis 26: 4751-4758, 2005.

30. Engbring JA and Kleinman HK: The basement membrane matrix in malignancy. J Pathol 200: 465-470, 2003.

31. Kleinman HK, Philp D and Hoffman MP: Role of the extracellular matrix in morphogenesis. Curr Opin Biotechnol 14: 526-532, 2003.
32. Ponce ML, Nomizu M, Delgado MC, Kuratomi Y,Hoffman MP, Powell S, Yamada Y, Kleinman HK and Malinda KM: Identification of endothelial cell binding sites on the laminin gamma 1 chain. Circ Res 84: 688-694, 1999.

33. Wang Y, Gong C, Yang L, Wu Q, Shi S, Shi H, Qian Z and Wei Y: 5-FU-hydrogel inhibits colorectal peritoneal carcinomatosis and tumor growth in mice. BMC Cancer 10: 402, 2010.

34. Yu L, Wang Y, Yao Y, Li W, Lai Q, Li J, Zhou Y and Kang T: Eradication of growth of HER2-positive ovarian cancer with trastuzumab-DM1, an antibody-cytotoxic drug conjugate in mouse xenograft model. Int J Gynecol Cancer 24: 1158-1164, 2014. 